Full citation: Novembre, G., Sammler, D., \& Keller, P. E. (2016). Neural alpha oscillations index the balance between self-other integration and segregation in real-time joint action. Neuropsychologia, 89, 414-425.

\title{
Neural alpha oscillations index the balance between self-other integration and segregation in real-time joint action.
}

Giacomo Novembre ${ }^{1,2,}{ }^{*}$, Daniela Sammler ${ }^{2}$, Peter E. Keller ${ }^{1}$

${ }^{1}$ The Marcs Institute for Brain, Behaviour and Development, Western Sydney University, Australia.

2 Max Planck Institute for Human Cognitive and Brain Sciences, Leipzig, Germany.

\section{Running title:}

Neural integration and segregation of self and other

* Corresponding author:

Dr. Giacomo Novembre

University College London.

Department of Neuroscience, Physiology and Pharmacology.

Gower Street, London, WC1E 6BT, UK

+44(0) 2076792156

g.novembre@ucl.ac.uk 


\begin{abstract}
Shared knowledge and interpersonal coordination are prerequisites for most forms of social behavior. Influential approaches to joint action have conceptualized these capacities in relation to the separate constructs of corepresentation (knowledge) and self-other entrainment (coordination). Here we investigated how brain mechanisms involved in co-representation and entrainment interact to support joint action. To do so, we used a musical joint action paradigm to show that the neural mechanisms underlying corepresentation and self-other entrainment are linked via a process - indexed by EEG alpha oscillations - regulating the balance between self-other integration and segregation in real time. Pairs of pianists performed short musical items while action familiarity and interpersonal (behavioral) synchronization accuracy were manipulated in a factorial design. Action familiarity referred to whether or not pianists had rehearsed the musical material performed by the other beforehand. Interpersonal synchronization was manipulated via congruent or incongruent tempo change instructions that biased performance timing towards the impending, new tempo. It was observed that, when pianists were familiar with each other's parts, millisecond variations in interpersonal synchronized behavior were associated with a modulation of alpha power over right centroparietal scalp regions. Specifically, high behavioral entrainment was associated with self-other integration, as indexed by alpha suppression. Conversely, low behavioral entrainment encouraged reliance on internal knowledge and thus led to self-other segregation, indexed by alpha enhancement. These findings suggest that alpha oscillations index the processing of information about self and other depending on the compatibility of internal knowledge and external (environmental) events at finely resolved timescales.
\end{abstract}

Keywords: Alpha oscillations, co-representation, joint action, music, self-other entrainment. 


\section{INTRODUCTION}

Interpersonal coordination and shared knowledge are two critical building blocks of social behavior. These constructs make up the foundation upon which most of our everyday interactions are built, and from which complex behaviors, including verbal and non-verbal communication, might have evolved (Greenfield 1994; Tomasello et al. 2005; Merker et al. 2009). Whether social interactions are possible depends on the brain capacity to integrate (internal) self-generated actions with the (external) motor output of other individuals while maintaining a distinction between self and other in order to achieve autonomous control of one's actions. This capacity for simultaneous action control (related to the Self), action monitoring (related to Others), and self-other integration and segregation have been mostly investigated with respect to two constructs: self-other entrainment (Tognoli et al. 2007; Dumas et al. 2010; Naeem et al. 2012a) and corepresentation (Sebanz et al. 2003; Sebanz, Bekkering, et al. 2006; Knoblich and Sebanz 2008). These constructs originate from dynamical vs. cognitive systems approaches to social interaction, respectively, which are associated with physically based vs. mental phenomena.

Self-other entrainment, defined broadly, involves the synchronization or spatiotemporal coordination of actions within a group of individuals having a social or communicative function (Clayton et al. 2005; Phillips-Silver and Keller 2012; Cacioppo et al. 2014; Keller et al. 2014). Self-other entrainment, in humans as in other species such as fireflies, crickets, and frogs (Merker 2000), underlies elementary (often spontaneous) forms of social behavior that might be 
ultimately important for the survival of a group and for reproduction (Greenfield 1994; Merker et al. 2009). In humans, interpersonal entrainment is observable in several kinds of social interactions, from walking with another individual to team sport and musical or dance ensembles (McGarry et al. 2002; van Ulzen et al. 2008; Keller et al. 2014). Self-other entrainment increases social affiliation (Hove and Risen 2009; Wiltermuth and Heath 2009), prosocial behavior (Kirschner and Tomasello 2010; Kokal et al. 2011; Cirelli et al. 2014), and is impaired in a variety of social disorders including schizophrenia, social anxiety and autism (Varlet et al. 2012, 2014; Marsh et al. 2013).

The mechanisms that support interpersonal entrainment have been interrogated in sensorimotor synchronization experiments requiring individuals to coordinate simple movements (e.g., finger taps) with external pacing sequences (generated by a computer or another individual). Such entrainment phenomena can be described using both linear (information processing) and nonlinear (coupled oscillator) dynamical approaches (Schmidt et al. 1990; Vorberg and Wing 1996; Repp 2005; Marsh et al. 2006; Richardson et al. 2007). The former work has revealed that sensorimotor synchronization is underpinned by temporal error correction and continuous coupling processes that enable internal timekeepers-oscillations of neural populations in co-actors' brains-to remain entrained with rhythms in the external environment (Repp 2005; Large 2008; Repp and Su 2013). Behavioral experiments and computational modeling have shown that the accuracy of synchronization is determined by properties of the error correction processes that modulate the coupling strength of the timekeepers to the external signal, as well as the degree of mutual adaptation 
between interacting partners (Repp 2005; Repp and Su 2013; van der Steen and Keller 2013; van der Steen et al. 2015).

The co-representational approach (Sebanz et al. 2003; Sebanz, Bekkering, et al. 2006; Sebanz, Knoblich, et al. 2006), on the other hand, provides a cognitive account of how individuals cooperate by pursuing shared task goals during joint action. This approach draws on common coding (Prinz 1997, 2013) and mirror mechanism theories (Rizzolatti and Sinigaglia 2010) that postulate an overlap in functional processes and neuroanatomical structures involved in perception and action. The co-representational approach holds that individuals internally simulate actions executed by an interaction partner and that this simulation is integrated with representations of one's own action goals during joint action (Newman-Norlund et al. 2007; Bekkering et al. 2009). Compared to self-other entrainment, co-representation is a higher-level cognitive resource that relies on internalized knowledge and sensorimotor experience with a given task (CalvoMerino et al. 2005, 2006; D'Ausilio et al. 2006; Lahav et al. 2007). For instance, motor familiarity with the actions performed by a partner has been found to strengthen co-representation (Hadley et al. 2015; Novembre et al. 2012, 2014; Loehr et al. 2013; Ragert et al. 2013). This process is likely to enable anticipatory motor simulations of others' actions (Kilner et al. 2007; Aglioti et al. 2008; Urgesi et al. 2010) that are based upon internal models. These models are acquired through sensorimotor experience and come to represent transformations between action goals, motor processes, and the sensory consequences of action execution (Wolpert et al. 2003; Lee and Noppeney 2011; Keller 2012; Keller et al. 2014). Through action simulation, an individual co-represents and anticipates 
others' actions by means of his or her own action repertoire (Wilson and Knoblich 2005; Knoblich and Sebanz 2008).

Self-other entrainment and co-representation are possibly the two most influential theoretical approaches to the study of joint action. However, these approaches appear to be conceptually different to the extent that they originate from physical (entrainment) vs. psychological (co-representation) theories of cognition. While the notion of entrainment assumes a dynamical coupling between an organism and the surrounding (external) environment, corepresentation focuses on mental (internal) representations. The internal vs. external focus of these approaches has led to debate concerning the relationship between self-other entrainment and co-representation and their underling mechanisms (Pressing 1999; Krampe et al. 2002; Knoblich and Sebanz 2008; Kaplan and Bechtel 2011; Schmidt et al. 2011; Stepp et al. 2011; Coey et al. 2012; Colling and Williamson 2014; Cummins 2014).

Here we propose that the mechanisms underlying real-time entrainment and corepresentation might be linked via a neural process that regulates the balance between self-other integration and segregation. We reasoned that the regulatory function of this neural process entails orchestrating the use of internal (knowledge) and external (environmental) sources of information during interpersonal coordination. We identified a putative marker of this process in neural oscillations within the alpha frequency band $(7-13 \mathrm{~Hz})$. Modulations of the alpha rhythm have been consistently reported to be implicated both in selfother entrainment tasks associated with interpersonal coordination (Tognoli et 
al. 2007; Dumas et al. 2010; Naeem et al. 2012a, 2012b; Konvalinka et al. 2014) as well as during action observation (Gastaut, H. J., \& Bert 1954; Hari et al. 1998; Caetano et al. 2007; Arnstein et al. 2011), especially if the viewer has motor experience with the observed action (van Elk et al. 2008; Cannon et al. 2014; Quandt and Marshall 2014). In particular, the lower alpha band (i.e. 8-10 Hz, as opposed to upper alpha between $10-12 \mathrm{~Hz}$ ) has been implicated in action observation (Frenkel-Toledo et al. 2014). Suppression of oscillations within the alpha frequency range is an index of cortical excitability (Tamura et al. 2005; Sauseng et al. 2009) and enhanced efficiency of cognitive processing (Klimesch 2012). Alpha oscillations are a promising candidate for linking entrainment and co-representation, as these oscillations originate from multiple brain regions including somatosensory (Salmelin and Hari 1994), motor (Arnstein et al. 2011) and parietal areas (Samaha et al. 2015), and their suppression has intrinsic temporal anticipatory properties (Nobre 2001; van Ede et al. 2011) that are relevant for integrating perception and action (Rohenkohl and Nobre 2011).

In the present study, we hypothesized that alpha oscillations associated with sensorimotor functions reflect the neural process that balances the use of internal (knowledge) and external (environmental) sources of information during joint action. We tested this hypothesis using a musical joint action task (cf. Novembre et al. 2012, 2014) that involved pairs of pianists performing complementary musical phrases (see Figure 1). Musical duet performance is a domain that provides a fair testing ground for examining the relationship between self-other entrainment and co-representation in an ecological yet experimentally controlled scenario (cf. DAusilio et al. 2015) (see also Schilbach 
2010; Acquadro et al. 2016). Employing a musical paradigm is appropriate given that joint musical performance is considered to be one of the oldest forms of social collaborative behavior (Mithen et al. 2006), and one where both interpersonal synchrony and co-representation come into play (Keller et al. 2014). Indeed, musical interaction can be viewed as a representative case of joint action where internal and external information are commensurate in importance and must be balanced. On this view, the members of a musical ensemble simultaneously access internal representations of an intended goal (e.g. the musical piece to be performed), while monitoring others' ongoing performances and adapting accordingly, possibly even deviating from the original intended goal when the need arises (Keller et al., in press).

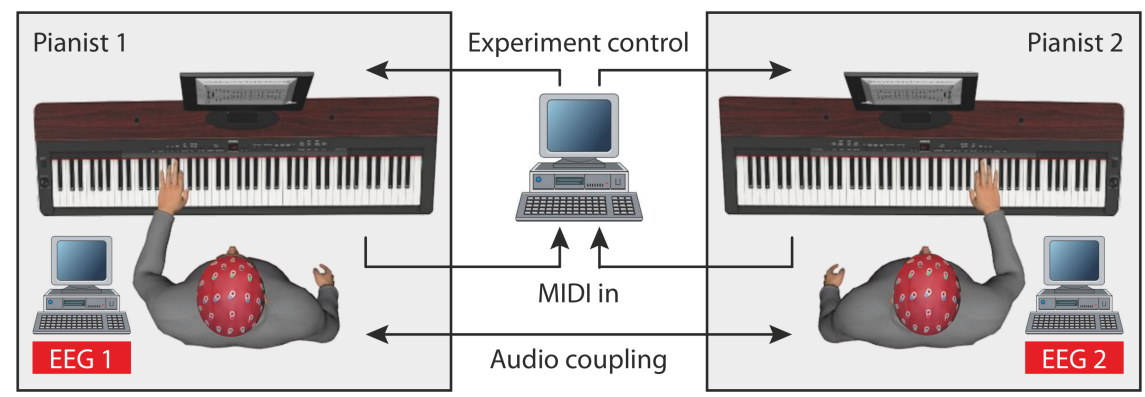

Figure 1: Illustration of the experimental setting. Two pianists, forming a pair, performed two complementary musical parts in two separate shielded booths. Behavioral performance (relative timing of the keystrokes) and EEG were recorded simultaneously.

Our musical paradigm allowed us to manipulate the external and internal focus of co-performers, i.e. self-other entrainment and co-representation, as independent factors while measuring alpha power in the EEG. It was assumed that an interactive effect of these two factors on alpha power would constitute evidence for a neural process linking self-other entrainment and co- 
representation. Alternatively, main effects of the two factors (in the absence of an interaction) would suggest that self-other entrainment and co-representation are independent.

In order to modulate the level of self-other entrainment, the paired pianists were instructed to jointly perform musical items comprising two musical phrases separated by a pause. The first phrase was performed at a fixed tempo (120 beats per minutes, bpm), while the second phrase was either faster or slower (150 or $96 \mathrm{bpm}$ ) in accordance with task instructions. These instructions were given (via a visual cue) at the start of the trial (i.e., prior to initiation of the first phrase), and it was therefore assumed that each pianist's internal timekeeper would be biased towards the new - impending - tempo from the outset. It was further assumed that this bias would lead to anticipatory tempo change behaviors at the millisecond time scale already in the first phrase (Repp 2001; Repp and Keller 2004). We manipulated the congruence of these anticipatory tempo changes (see Figure 2) by providing the two pianists with either the same TEMPO instruction (both $150 \mathrm{bpm}$ or $96 \mathrm{bpm}$ ) or different instructions (one pianist $150 \mathrm{bpm}$ and the other $96 \mathrm{bpm}$ ).

It was expected that self-other entrainment, and hence interpersonal synchrony, during performance of the first phrase would be higher in congruent than incongruent tempo conditions (due to the impending tempo change being the same across pianists for the former, see Figure 2). The accuracy of interpersonal synchrony was quantified by computing the degree of asynchrony between the timing of paired pianists' complementary keystrokes. Furthermore, we 
quantified the relative influence of each pianist's timing upon the other's timing, i.e. coupling strength and mutual adaptation (see methods).

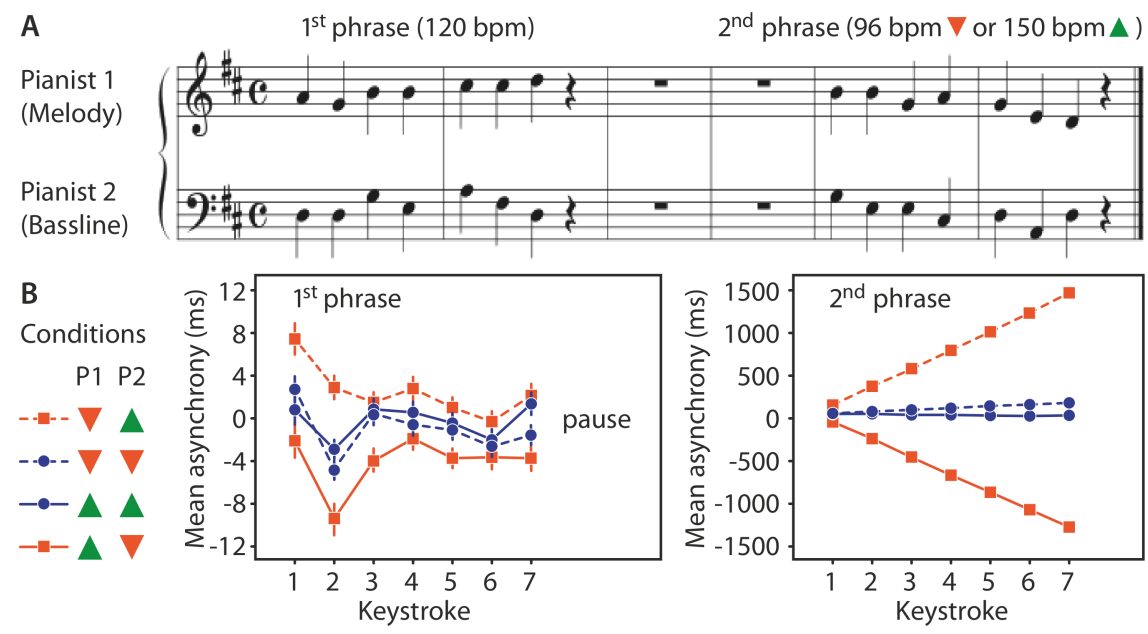

Figure 2: A: Example of a musical score played by two pianists on digital pianos. One pianist performed the melody with the right hand while the other performed the bassline with the left hand. Performance tempo was $120 \mathrm{bpm}$ for the initial two bars (first phrase), and either 96 or $150 \mathrm{bpm}$ for the last two bars (second phrase). The pianists could not hear each other in the second phrase. Behavioral and EEG data presented below are associated with the first phrase. B (left): Visual cues, presented to pianist 1 (P1) and 2 (P2) before performance of the first phrase, instructing the tempo to be played in the second phrase. B (center): Mean asynchronies associated with the first phrase, across 4 tempo instructions. B (right): Mean asynchronies associated with the second phrase. The divergence in asynchronies in the tempo incongruent conditions (red squares) indicates that pianists anticipated the instructed tempo change during the first phrase and executed the change accurately in the second phrase (note the different time scale between center and right B panels). The manipulation thus served to enhance interpersonal synchronization, at millisecond timescale, during performance of the first phrase in the tempo congruent conditions. The behavioral and EEG results reported below are associated with performance of the first musical phrase. Error bars are one standard error of all asynchronies across pairs and trials. 
To address co-representation, the degree to which co-performers were familiar with each other's part was manipulated by allowing the pianists to practice just their own part or both pianists' parts prior to the experiment. Consistently with previous studies, action FAMILIARITY with the other player's part was intended to boost co-representation by recruiting motor representations associated with the performer's action repertoire (Novembre et al. 2012, 2014; Loehr et al. 2013; Ragert et al. 2013; Hadley et al. 2015). It was expected that familiarity would thus encourage greater reliance upon internal sources of information (knowledge of both parts) rather than full reliance upon external sources of information (perceptual input from the co-performer).

In operational terms, behavioral (coupling strength and mutual adaptation) and electrophysiological (EEG alpha power) measures were assessed in a TEMPO $\mathrm{x}$ FAMILIARITY factorial design. Mutual adaptation was expected to change as a function of TEMPO, with congruent tempi leading to higher external focus and increased self-other mutual adaptation. Action FAMILIARITY was conversely expected to weaken coupling strength by encouraging the pianists to rely more strongly on internal representations rather than the actual performance of their partner (cf. Keller et al., 2007, Ragert et al. 2013). Crucially, to the extent that alpha oscillations regulate the balance between internal (knowledge) and external (environmental) sources of information during interpersonal coordination, we predicted that our analysis of alpha power would yield an interaction between TEMPO and FAMILIARITY. Given the integrative function of 
alpha suppression discussed above, we expected that the strongest suppression should be observed when internal and external sources of information are highly compatible. This compatibility should be highest in the familiar condition when tempo is congruent, i.e. when internal and external sources of information are most similar. To measure this effect, we examined how brain activity in each individual pianist changes as a function of self-other entrainment and corepresentation during joint performance of the first musical phrase (see Figure 2).

\section{MATERIALS and METHODS}

\subsection{Participants}

Twenty-eight amateur pianists (aged 19-33, 9 males, 1 left handed) were recruited individually to participate in this study. The pianists were paired irrespectively of gender (resulting in 6 pairs composed only of female players, 1 of only males, and 7 mixed pairs) or age (the mean age difference between pianists was 3.42 years, s.d. $=3.69$ ), forming 14 pairs. Mean years of piano training of each pair ranged between 9 and 21 years (mean=13.42, sd=3.61). Participants were naïve with regard to the purpose of the study. They had no neurological or psychiatric history, and had normal or corrected to normal vision. All pianists provided written informed consent and were paid for their participation. This study was approved by the local ethics committee of the University of Leipzig and was conducted according to the declaration of Helsinki. 


\subsection{Musical material and pre-experimental training}

One week prior to the experiment, individuals in each pair received musical scores of 4 excerpts from chorales by J. S. Bach (cf. Novembre et al. 2012, 2014). Each chorale excerpt consisted of 2 musical phrases, each 2 bars in length containing 7 quarter notes followed by a quarter note rest. Two silent 'rest' bars were inserted between the two phrases, giving a total of 6 bars in each score, see Figure 2.

Co-representation was manipulated by varying pianists' familiarity with the musical part performed by the co-performer. Pianists learned both the melody (with the right hand) and the bassline part (with the left hand) for two out of the four scores, while they only learned the melody or bassline (complementarily within each pair) for the remaining two scores. The scores for which both parts were learned were counterbalanced across two groups of pairs $(n=7)$, i.e. one group of pianists learned both parts from musical scores A and B, but only one part from score C and D, while another group did the opposite.

Different degrees of self-other entrainment were induced by manipulating the congruence between co-performers' tempi. The pianists were instructed to practice the initial two bars of each score at a tempo of 120 beats per minutes (bpm), and the final 2 bars at a faster (150 bpm) and a slower (96 bpm) tempo (see Figure 2). To assist the pianists in learning the correct tempi, they were instructed to practice the material in time with audio recordings of a metronome beating at the target tempo. 
The performance level of all pianists was assessed in a brief practice session at the beginning of the EEG experiment. Only pianists who were able to play all items fluently (i.e. without errors in pitch or timing) and to accurately perform the tempo changes continued the experimental session. (For one pair, the session was postponed by one day to allow sufficient practice.) Pianists could hear each other throughout the entire practice session (i.e. during both phrases), and only congruent tempo changes were employed.

\subsection{Experimental setting and procedure}

The two pianists in each pair were seated in two separate shielded booths and were therefore not in visual contact. Each booth contained a digital MIDI piano (Yamaha Clavinova CLP150) with headphones (Sennheiser HD 280 Professional), a monitor placed on top of the piano, an EEG amplifier, and a video camera (with aerial view) monitoring the pianists from the control room (where the experimenters were seated).

Each trial began with a fixation cross presented on each monitor (500 msec), followed by a visual cue (1000 msec) - either a green triangle pointing upward or a red triangle pointing downward (see Figure 2B) - indicating whether each pianist was supposed to play faster or slower in the final two bars. Each pianist was only presented with one cue, and was not aware of what cue the coperformer had been presented with. After the cue, a metronome playing four beats ( 1 bar) at $120 \mathrm{bpm}$ (beat duration $=500 \mathrm{msec}$ ) was presented to indicate 
when to start playing the 6-bar-long musical items. Bars 1 and 2 (comprising the first musical phrase, see Figure 2A) were played at a constant tempo of $120 \mathrm{bpm}$ and pianists could hear the mixed feedback (i.e. both parts combined) of the two digital pianos via headphones (the mixed parts were played through the left and right channels simultaneously). There was no music to play during bars 3 and 4, so the pianists waited (at the original tempo) until bars 5 and 6 . They played bars 5 and 6 at the new tempo (according to the initial visual cue), leading to congruent or incongruent new tempi between the co-performers. Crucially, during this stage both pianos were muted, i.e. the pianists could neither hear themselves nor the other pianist playing at the new tempo. This was intended to ensure that the pianists remained unaware of the tempo congruence manipulation throughout the experiment and to avoid tempo adaptation during the mute phase. Furthermore, both pianos (not only the other's piano) were muted to avoid giving the impression of performing the task individually. During the trial, each pianist saw the score of only his or her part on the monitor (the score disappeared at the point when performance was expected to end based on the new tempo).

Each pair completed 48 trials for each condition in the $2 \times 2$ factorial design, resulting in a total of 192 trials (each trial corresponded to the performance of one score). The factors were FAMILIARITY with the other pianist's part (familiar vs. unfamiliar) and TEMPO change in the last two bars (congruent vs. incongruent). The total duration of the experiment was about 60 minutes. All participants took a 10-minute break after half of the trials. 


\subsection{Behavioral and EEG recording}

A computer program written in Presentation software (Version 14.9, Neurobehavioral Systems, Inc.) controlled the display of the tempo cues, the scores and the presentation of the metronome (while sending triggers to two computers recording EEG). The computer received codes corresponding to the keystrokes from both pianos (for this purpose, the MIDI signals sent from the pianos were converted into serial codes by two custom-built interfaces). The program also controlled the audio-feedback provided to the pianists via a dedicated switch that cut off the feedback from the beginning of the third bar.

EEG was recorded from $29 \mathrm{Ag} / \mathrm{AgCl}$ electrodes mounted in an elastic cap according to the extended international 10-20 system (Sharbrough, F., Chatrian, G. E., Lesser, R. P., Lüders, H., Nuwer, M., \& Picton 1991). The electrode positions were: FP1, FPZ, FP2, F7, F3, FZ, F4, F8, FC5, FC1, FC2, FC6, T7, C3, CZ, C4, T8, CP5, CP1, CP2, CP6, P7, P3, PZ, P4, P8, 01, OZ, 02 (Dumas et al. 2010). Two mastoid electrodes (i.e. placed on the mastoid bones behind the ears) were also included. The left mastoid served as reference, while the average of left and right mastoids was used for off-line re-referencing. The ground electrode was located on the sternum. Horizontal and vertical electrooculograms were bipolarly recorded from electrodes placed on the outer canthus of each eye, as well as above and below the right eye. Impedances were kept below $5 \mathrm{k} \Omega$. Signals were amplified with one 24 bit Brainvision QuickAmp 27 amplifier per pianist (Brain Products GmbH, Gilching, Germany) and digitized with a sampling rate of $500 \mathrm{~Hz}$. Data were recorded with in-house software (Qrefa, EEP 3.2, commercially available as 
eeprobe $^{\mathrm{TM}}$ from ANT neuro, www.ant-neuro.com) and stored on two separate PCs with identical hardware.

\subsection{Data analysis}

\subsubsection{Behavioral}

Interpersonal synchronization accuracy, coupling strength and mutual adaptation were quantified by analyzing pianists' performance on the digital instruments (keystroke time). For this, we computed the pianists' keystroke asynchronies (elapsed time between complementary keystrokes) and crosscorrelations of the inter-keystroke-intervals (IKIs).

Trials in which at least one pianist made a pitch mistake (7.9\% of all trials) or the pianists did not change tempo as instructed (5.7\% of all trials) were discarded from further analyzes. Tempo errors were identified by fitting the difference of the actual IKIs (pianist 1 IKI - pianist 2 IKI) to the difference values that would be expected following correct performance of congruent or incongruent tempi in the final two bars. The goodness of fit for the regression model of the mean squared error was computed. Trials that were best predicted by tempi other than the instructed ones were considered erroneous.

Each pair's synchronization was assessed by analyzing keystroke asynchronies (Keller et al. 2007; Ragert et al. 2013). Mean asynchrony for each position (i.e. note position within the musical phrase) can be seen in Figure 2B. Before 
averaging asynchronies across positions, we normalized these values by subtracting the mean asynchrony (computed for each position) from the actual data (separately for each of the four musical items (Wing et al. 2014)) to account for variations in asynchrony related to different metric positions (Keller 2014). Next, participants' normalized asynchronies were averaged across items, transformed into absolute values, and entered into a $2 \times 2$ repeated measures ANOVA with factors TEMPO (congruent, incongruent) and FAMILIARITY (familiar, unfamiliar).

Inter-Keystroke-Intervals (IKIs) were used to assess coupling strength and mutual adaptation (cf. Goebl and Palmer 2009; Konvalinka et al. 2010, 2014). The series of 6 intervals associated with 7 keystrokes in each trial (within each musical phrase) were used to compute cross-correlation coefficients at lag 1 (comprising the averaged lags +1 and -1 ) and lag 0 . The averaged coefficients (Fisher z-transformed) were entered into two separate $2 \times 2$ repeated measures ANOVAs with factors TEMPO (congruent, incongruent) and FAMILIARITY (familiar, unfamiliar).

\subsubsection{Electroencephalogram (EEG)}

EEG data were analyzed using FieldTrip (downloaded on 2012-05-12) toolbox (Oostenveld et al. 2011) in Matlab. Raw EEG data were high- and low-pass filtered (cutoff frequencies: $0.4 \mathrm{~Hz}$ and $45 \mathrm{~Hz}$, Butterworth, filter-order: 3) and re-referenced to linked mastoids. EEG signals were epoched from $-3 \mathrm{~s}$ to $+13 \mathrm{~s}$ relative to the onsets of performance (comprising the visual cue, metronome, 
and the performance time $+1 \mathrm{~s}$ as buffer for the FFT). EEG data associated with behaviorally incorrect trials (cf. behavioral analysis) were discarded. Time points containing technical and strong muscle artifacts (e.g., due to coughing or jaw movements) were rejected following identification through visual inspection. Sections rejected in one pianist were also rejected in the other pianist. Overall, $20.34 \%$ of all the trials were rejected according to behavioral (see above) and EEG criteria, leaving an average of 152.92 trials per pair (s.d. 16.19). In a second step, the artifact-reduced and (behaviorally) error-free data were entered into an Independent Component Analysis (ICA, InfoMax), and components representing eye movements or other movement-related activity, including continuous tension of muscles (e.g. at frontal electrodes), and slow drifts were rejected.

Artifact-free neuroelectric time series were submitted to fast Fourier transformation. For each of the four conditions (familiar congruent, familiar incongruent, unfamiliar congruent, unfamiliar incongruent), time-frequency series were computed from $-1000 \mathrm{~ms}$ to $+5000 \mathrm{~ms}$ relative to the onset of performance of the initial or final part of the musical items using a Hanningtapered window with 5 cycles and a 100 ms time resolution (using the 'ft_freqanalysis' function with 'mtmconvol' method as implemented in FieldTrip). Power estimates were calculated for frequencies ( $1 \mathrm{~Hz}$ bins) between 2 and 40 Hz. Statistics were performed on the alpha band, the range of which was defined as $\pm 2 \mathrm{~Hz}$ centered around the individual alpha peak of each pianist (mean: 9.96 $\pm 1.62 \mathrm{~Hz}$, range: 7-13 Hz) at electrode Cz (Klimesch 1997; Bazanova and Vernon 2013). Further analyses assessed the specific contribution of low and high alpha sub-bands, spanning the $2 \mathrm{~Hz}$ range below or above the individual peak 
frequency, respectively (Tognoli et al. 2007; Naeem et al. 2012a; Dumas et al. 2014; Frenkel-Toledo et al. 2014).

Individual datasets were normalized similarly to previous EEG (Meyer et al. 2011) and MEG (van Ede et al. 2010) studies. A normalization procedure was preferred over baseline correction because metronome sounds were presented prior to our epochs, which led to noticeable modulations of power across all frequencies. Specifically, for each participant, the average band power across $N=$ 4 conditions (C) was subtracted at each electrode (e), frequency (f), and time bin (t) from the individual (condition specific) band power. This difference value was further divided by the average band power to center values around zero.

$$
\hat{P}_{C(e, f, t)}=\frac{P_{C(e, f, t)}-1 / N * \sum_{C=1}^{N} P_{C(e, f, t)}}{1 / N * \sum_{C=1}^{N} P_{C(e, f, t)}}
$$

For statistical analyzes, the (within-participant) normalized data from each pair of pianists were averaged. This was done to 1) equate sample size and thus statistical power across behavioral and EEG analyses, and 2) to meet the requirement of parametric statistical tests of independent data points, which would otherwise be violated given that two pianists from the same pair cannot be assumed to be independent ${ }^{1}$.

Mean alpha power from $0 \mathrm{~ms}$ to $+4000 \mathrm{~ms}$ relative to the onset of performance in the first phrase was compared between conditions. In accordance with the $2 \times 2$

\footnotetext{
1 We ran additional analyses to confirm that equivalent results to those reported here are obtained if data are not normalized or averaged within pairs of pianists.
} 
factorial design, the main effects of TEMPO, FAMILIARITY and the interaction between TEMPO $\times$ FAMILIARITY, were computed using a cluster-based nonparametric permutation test (cf. Maris and Oostenveld 2007), which controls for false-alarm rate by using a cluster statistic that is evaluated under a single permutation distribution. More specifically, this algorithm compares alpha power between conditions, separately for each channel, using paired-samples ttests, yielding one t-value for each channel. The algorithm then forms clusters of neighboring channels (based on the minimum neighborhood distance) whose tvalues exceed the significance criterion (here $\mathrm{p}<0.05$ ) and computes a clusterlevel statistic value (sum of t-values within the cluster). Next, cluster-level statistics from 1000 random partitions of the data are used to build a distribution upon which the significance probability (here $\mathrm{p}<0.05$ ) of the experimental cluster can be estimated.

In order to run this analysis in the Fieldtrip toolbox (see above), it was necessary to average data across conditions to assess the main effects and interactions using t-tests. Main effects were tested by contrasting the averaged familiar vs. unfamiliar conditions (irrespective of tempo congruence) or congruent vs. incongruent tempo conditions (irrespective of familiarity). The interaction was tested by contrasting the averaged familiar-congruent and unfamiliarincongruent conditions vs. the averaged familiar-incongruent and unfamiliarcongruent conditions.

\section{RESULTS}


We first report results concerning the effects of TEMPO congruence and FAMILIARITY on behavioral measures of interpersonal coordination during performance of the first musical phrase. Following that, we present results concerning the effects of these factors on EEG alpha suppression intraindividually.

\subsection{Behavioral results}

Behavioral measures of interpersonal coordination included indices of synchronization accuracy, the strength of interpersonal coupling, and mutual adaptation (see Figures 2 and 3).

\subsubsection{Interpersonal synchronization accuracy}

Synchronization accuracy was estimated by computing asynchronies, i.e. elapsed time between complementary keystrokes produced by the two pianists (see methods). Normalized mean absolute asynchronies, averaged across pairs, are displayed in Figure 3A. Mean asynchrony data were entered into a 2x2 ANOVA with within-subject factors TEMPO (congruent vs. incongruent) and FAMILIARITY (familiar vs. unfamiliar). The ANOVA yielded a significant main effect of TEMPO $(\mathrm{F} 1,13=24.89, \mathrm{p}<.001)$, while the main effect of FAMILIARITY and the TEMPO x FAMILIARITY interaction were not significant (all Fs $<1.1$, all ps>.1). The main effect of TEMPO indicated that interpersonal synchronization was higher in congruent than incongruent conditions. Thus, the pianists were more synchronized when they were planning the same (congruent) tempo 
change than different (incongruent) tempo changes. This suggests that pianists were already anticipating the tempo to be produced during the second musical phrase while they were performing the first phrase (see Figure 2).
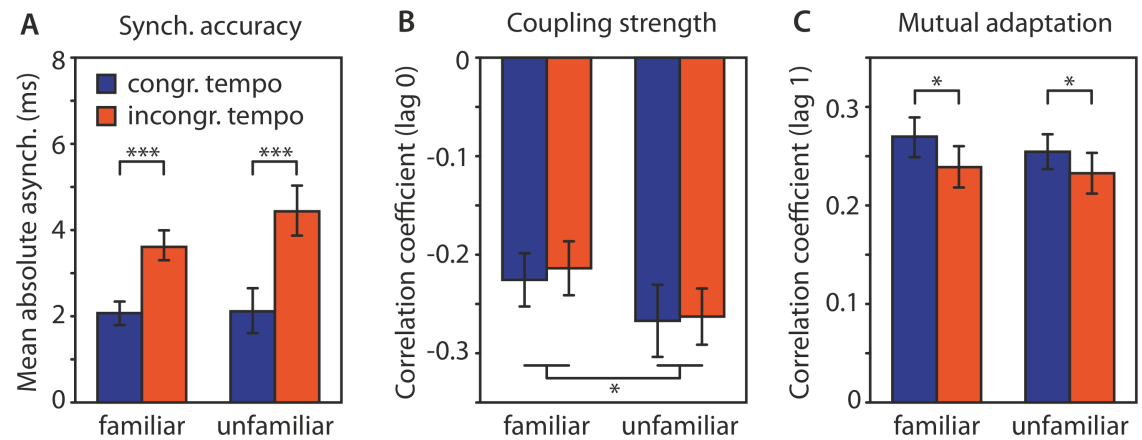

Figure 3: Behavioral measures of synchronization accuracy (A: Mean absolute asynchronies), coupling strength (B: Lag zero cross correlation coefficients) and mutual adaptation (C: Lag 1 cross correlation coefficients) computed across TEMPO and FAMILIARITY conditions. Error bars are $1 \mathrm{SE}$ of the mean.

\subsubsection{Coupling strength \& mutual adaptation}

Measures of the strength of interpersonal coupling and mutual adaptation were obtained by computing the cross correlation coefficients of paired pianists' interkeystroke intervals at different lags (see Methods). Lag 0 cross correlations were taken as an index of coupling strength (i.e., the degree to which fluctuations in local tempo were matched across pianists). Previous computational modeling work has shown that, as coupling strength increases, the lag 0 cross-correlation coefficient becomes more negative in value (Vorberg and Wing 1996). Lag 1 cross correlations indexed the influence of each pianist's timing upon the other's timing (i.e., the degree to which pianists copied each other's timing deviations 
from one inter-keystroke interval to the next). As mutual influence increases, the lag 1 cross-correlation coefficient becomes more positive (Konvalinka et al. 2010).

Results for co-performer coupling strength, as indexed by the lag 0 crosscorrelation of inter-keystroke intervals, are displayed in Figure 3B. An ANOVA on these data yielded a significant main effect of FAMILIARITY (F1,13=5.050, $\mathrm{p}=.043$ ), indicating that, when the pianists had practiced the other's part, lag 0 coefficients were closer to zero (i.e., less negative). This suggests that the timing of the performances was less well matched when the pianists were familiar with each other's actions. In other words, co-representation (increased by familiarity) weakened the strength of the coupling between pianists' keystrokes (cf. Keller et al. 2007; Ragert et al. 2013). The main effect of TEMPO and the TEMPO $\mathrm{x}$ FAMILIARITY interaction were not significant (all Fs $<1$, all $p>.1$ ).

Results for mutual adaptation, indexed by the lag 1 cross correlation of interkeystroke intervals, are displayed in Figure 3C. An ANOVA on these data yielded a significant main effect of TEMPO (F1,13=5.057, $\mathrm{p}=.042)$, indicating that the pianists influenced each other to a stronger degree in TEMPO congruent conditions. This suggests that preparing for a congruent tempo change induced higher mutual adaptation between the pianists, which is consistent with the synchronization accuracy data showing that co-performers were more entrained in TEMPO congruent conditions. The main effect of FAMILIARITY and the TEMPO x FAMILIARITY interaction were not significant (all Fs $<1$, all ps $>$.1). 
In sum, the behavioral results suggest that co-representation (associated with familiarity with the co-performer's part) weakened interpersonal coupling while self-other entrainment (facilitated by tempo congruence) increased mutual adaptation between co-performers.

\subsection{EEG results}

The analyses of the EEG data provided condition-specific power spectra associated with performance of the first musical phrase (i.e. where the behavioral results discussed above were observed, see Figure 2). This measure reflects neural processes at the level of individual brains within pairs of coperformers. We tested for electrode clusters exhibiting effects of TEMPO congruence, FAMILIARITY, and the interaction of these factors in separate

permutation analyses (cf. Maris and Oostenveld 2007), by analyzing the normalized power in the individually defined alpha band (averaged for each pair of pianists) (see data analysis above).

\subsubsection{TEMPO congruence suppresses alpha power.}

We identified a cluster of electrodes with central-posterior topography that displayed a significant effect of TEMPO ( $p=.009$, electrodes: C3, CZ, C4, CP5, CP1, CP2, CP6, P3, PZ, P4, P8), reflecting stronger suppression of alpha power during tempo congruent than incongruent trials (Figure 4A). This result indicates that subtle differences in self-other synchronization and mutual adaptation (see Figures 2 and $3 \mathrm{~A}, \mathrm{C}$ ) were associated with a modulation of neural oscillations in 
the alpha band, with stronger alpha suppression being related to higher behavioral synchronization and greater mutual adaptation.
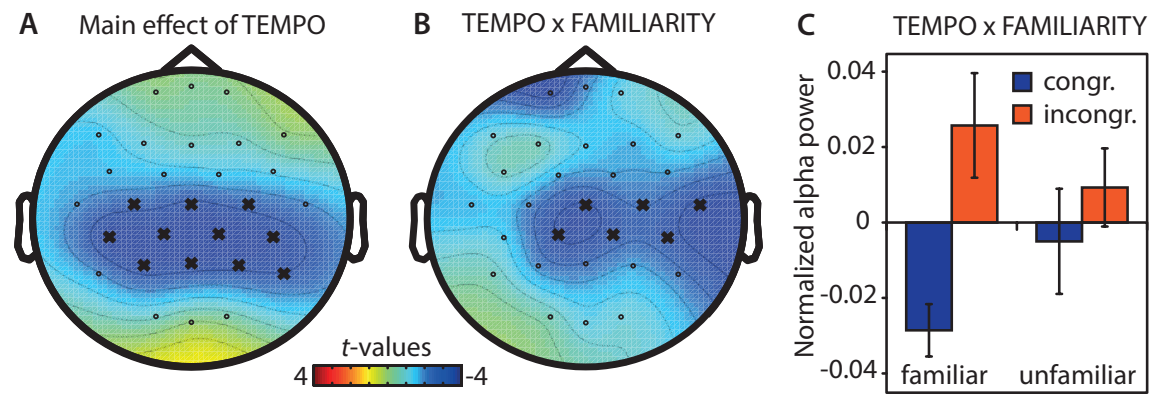

Figure 4: Topography of normalized alpha power for the main effect of TEMPO (A), and the interaction between TEMPO and FAMILIARITY (B). The electrodes associated with significant differences are marked by an ' $\mathrm{x}$ '. C: Normalized alpha power estimates associated with the cluster highlighted on the B panel, separately for Tempo and Familiarity conditions. Error bars are $1 \mathrm{SE}$ of the mean.

\subsubsection{FAMILIARITY modulates alpha power depending on TEMPO congruence.}

While no electrode clusters showed significant effects of FAMILIARITY, a cluster over right central and posterior regions displayed a significant interaction between TEMPO and FAMILIARITY (see Figure 4B, $p=.030$, electrodes: CZ, C4, T8, CP1, CP2, CP6). This interaction indicates that the effect of TEMPO on alpha power was dependent on FAMILIARITY. More specifically, higher or lower selfother entrainment (i.e. tempo congruence vs. incongruence) led to relative alpha suppression or enhancement, respectively, but only when the pianists were familiar, not when they were unfamiliar with each others' parts (see Figure 4C). 


\subsubsection{Lower (Alpha) band specificity.}

The latter analysis, testing the interaction between TEMPO and FAMILIARITY, was repeated with focus on lower and upper alpha bands (see methods). These analyses replicated the interaction between TEMPO and FAMILIARITY $(p<.040$, with topography as shown in Figure 4B) only when the lower, but not the higher, alpha band was selected (lower and higher alpha bands spanned $2 \mathrm{~Hz}$ below or above the individual's alpha peak).

\subsubsection{Alpha band specificity and dependence on auditory feedback}

Our effects did not extend to frequencies other than alpha and were dependent on auditory feedback (which was the sole means for social interaction; see Methods). Specifically, the analysis of power in other frequency bands, including theta $(4-7 \mathrm{~Hz})$, beta $(13-30 \mathrm{~Hz})$, and gamma $(30-40 \mathrm{~Hz})$, did not yield effects of TEMPO, FAMILIARITY, or their interaction. Furthermore, alpha power modulations were not observed during the performance of the second musical phrase (which was executed in silence).

\section{DISCUSSION}

This study used a musical joint action task to identify a neural process that, by balancing the use of internal vs. external information during joint action, links two fundamental mechanisms supporting social behavior: self-other 
entrainment and co-representation. The working of this process - indexed by EEG alpha oscillations - was associated with behavioral variations in self-other entrainment at the millisecond timescale. Alpha suppression was observed when self- and other-produced actions were strongly entrained in time. Crucially, we further show that alpha suppression was boosted when co-representation (action familiarity) was accompanied by high self-other entrainment. Conversely, a relative enhancement of alpha power was observed when co-representation was instead accompanied by small decrements in behavioral entrainment. Action familiarity thus led to alpha suppression or enhancement depending on the compatibility between internal knowledge and the external self-other relationship. Compatible internal and external relations (familiar actions and high self-other entrainment) favor integration and alpha suppression, whereas incompatible relations (familiar actions but low entrainment) favor segregation and alpha enhancement.

These findings suggest that, although the mechanisms underlying self-other entrainment and co-representation rely upon external vs. internal sources of information to differing degrees, these mechanisms are linked neurophysiologically in alpha oscillations. Specifically, this neurophysiological process regulates the balance between self-other integration and segregation depending on the compatibility of internal (knowledge) and external (environmental) information during social interaction.

\subsection{Behavioral effects of self-other entrainment and co-representation on interpersonal coordination.}


The effects of self-other entrainment and co-representation on behavioral measures of interpersonal coupling strength and mutual adaptation are informative about the real-time interaction between co-performers. On the one hand, high self-other entrainment was associated with greater mutual adaptation. This suggests that high levels of synchrony between paired pianists' sounds facilitated self-other integration and thus the timing of one performer's actions influenced the other's action timing to a greater degree. On the other hand, familiarity with the co-performer's part decreased interpersonal coupling strength, suggesting that co-representation lowered the degree to which fluctuations in timing were matched across pianists.

This pattern of results is consistent with a differential focus of self-other entrainment and co-representation on external vs. internal sources of information. While entrainment increases mutual adaptation between selfproduced actions and the effects of others' actions in the external environment, co-representation relies on internal knowledge and action simulation. To the extent that internal knowledge is grounded in an individual's own action repertoire, the timing of internally simulated actions might not match others' actual action timing, and hence co-representation decreases coupling strength (Keller et al. 2007; Ragert et al. 2013). This implies that co-representation decreases externally focused inter-individual coupling while increasing internally focused (intra-individual) processes, as indexed by EEG alpha oscillations (see next section). 
It is also worth mentioning that a considerable body of previous research has explored co-representation by means of the so-called joint Simon task (Sebanz et al. 2003), which has been criticized on the grounds that social constructs of self and other are potentially confounded with the spatial representation of response keys used in the experimental setup (Dolk et al. 2014). This particular criticism is not relevant to the present investigation because pianists were in separate rooms rather than side-by-side, they could not see each other, their sounds were mixed and played simultaneously through the left and right headphone channels, and the use of the left and right hand was counterbalanced.

\subsection{Interactive effects of self-other entrainment and co-representation on alpha oscillations.}

Neural oscillations in the alpha frequency band yielded evidence for an interaction between the mechanisms underpinning self-other entrainment and co-representation. Alpha suppression was strongest when co-representation (action familiarity) was accompanied by high self-other entrainment, while alpha enhancement occurred when co-representation was accompanied by lower entrainment. This interaction suggests that co-representation leads to a qualitative difference in the modulation of alpha power depending on the level of self-other entrainment. Familiarity potentially leads to the formation of a representation of another's action that is based upon internal models thatdrawing on an individual's own sensorimotor learning history-represent transformations between internal goals and the external sensory consequences of action (Loehr et al. 2013; Keller et al. 2014; Novembre and Keller 2014; 
Novembre et al. 2014; Loehr and Vesper 2015). The level of self-other entrainment then balances the use of these internal and external sources of information for the co-representation process. Thus, when interpersonal timing is highly synchronous (greater self-other entrainment), co-representation entails a more external (i.e. other-oriented) focus, resulting in higher self-other integration (Paladino et al. 2010; Colzato et al. 2012; Liepelt et al. 2012; Naeem et al. 2012a; Beckes et al. 2013; Novembre et al. 2014), as indexed by alpha suppression. When interpersonal timing is less synchronous, co-representation relies more upon internal knowledge (boosted by familiarity) and thus leads to self-other segregation (Ruby and Decety 2001; Brass et al. 2009; Dumas et al. 2012; Novembre et al. 2012), indexed by alpha enhancement.

We propose that alpha oscillations are a plausible candidate for regulating the balance between self-other integration and segregation during interpersonal coordination (although a causal role cannot be claimed, given the correlational nature of our results). Such a regulation process would involve balancing the use of internal (knowledge) and external (environmental) sources of information via alpha suppression and enhancement. Consistent with this view, the results of other research suggest that alpha suppression is associated with increased cortical excitability, reflecting the selective processing of task-relevant information (Jensen et al. 2012; Klimesch 2012) or, more generally, the access to and retrieval of information stored in a complex knowledge system across cognitive domains (Klimesch 1997, 2012). This excitability might be an index of temporal integrative processes (Klimesch et al. 2007; Jensen and Mazaheri 2010), which are facilitated when external information is congruent with internal cognitive and motor processes, as found in studies of speech 
intelligibility (Obleser and Weisz 2012) and movement coordination (Naeem et al. 2012a, 2012b). Alpha enhancement, on the other hand, has been linked to processes that serve to inhibit task-irrelevant information (Klimesch et al. 2007; Jensen and Mazaheri 2010), as demonstrated in studies of spatial attention (Worden et al. 2000; Thut et al. 2006), somatosensory working memory (Haegens et al. 2010), and long-term memory formation (Meeuwissen et al. 2011).

Although our findings provide evidence that self-other entrainment and corepresentation are linked, they also highlight the differential focus of entrainment on the external environment and co-representation on internal knowledge. This sets the scene for a potential tradeoff where the strengthening of co-representation within each individual's brain incurs the cost of reduced self-other coupling between the two individuals' behavior (Keller et al. 2007; Ragert et al. 2013). This tradeoff is managed by varying the degree of alpha suppression and enhancement in each individual to balance the detrimental effect of increased co-representation on interpersonal coupling against the facilitative effect of self-other entrainment on interpersonal synchrony and mutual adaptation.

The right centro-parietal topography of the observed interactive effect of corepresentation and self-other entrainment on alpha oscillations is consistent with an agency network that is responsible for relating one's body movements to observed movements produced by others (Brass et al. 2005, 2009). In line with our results, this agency network is typically right-lateralized as shown by 
multiple neuroimaging studies using EEG (Tognoli et al. 2007; Dumas et al. 2010; Naeem et al. 2012a), fMRI (Decety and Lamm 2007; Fairhurst et al. 2013, 2014), and brain stimulation methods (Tsakiris et al. 2008; Santiesteban et al. 2012). Note that these right-lateralized effects were observed while participants executed movements bimanually (Dumas et al. 2010) or with the right-hand (Tognoli et al. 2007; Dumas et al. 2010; Naeem et al. 2012a, Fairhurst et al. 2013, 2014). Together, these findings speak against a pure motor interpretation of the functions of such a network (which would instead predict either a bilateral or a classical contra-lateral effect in the left hemisphere), and are again in line with our study, where averaged right- and left-handed performances resulted in rightlateralized effects. Furthermore, the low-alpha band specificity of our effects buttresses our interpretation of an agency network operating to integrate or segregate representations of self and others because this sub-band has been linked specifically to action observation (Dumas et al. 2014; Frenkel-Toledo et al. 2014).

Note that we do not claim that the observed modulation of alpha power is necessarily specific to interactions that are "social". Rather, we believe that these modulations reflect general mechanisms that weight internal and external sources of information, which are highly relevant in social interactions but also apply to other forms of sensorimotor synchronization with non-human external signals. Indeed, the suppression of alpha oscillations, particularly over rightparietal regions, has been associated with attention-related processes that have internal focus (Benedek et al. 2014) and are involved in temporal prediction (Battelli et al. 2007; Samaha et al. 2015). This framework fits well with our 
results because the modulation of alpha power associated with self-other entrainment 1) had a right-centro-parietal topography and 2) was observed only during the familiar conditions, which according to the behavioral results were associated with a more internal focus. Our results thus potentially inform joint action accounts and theories that are concerned with whether and how attention fluctuates from self to others during the course of a joint musical performance (Schiavio and Hoffding 2015; Keller et al. 2016).

Finally, it is worth commenting on the extent to which our results might generalize beyond musical interaction and synchronous behavior. We believe that our findings are relevant to any form of interaction that requires real-time interpersonal coordination, whether it is musical or not. Indeed, other forms of coordination rely on internal representations and prior experience, which are likely to impact upon task performance. Furthermore, it can be noted that our participants were not classed as professional musicians, which makes generalizability more likely to the extent that extensive specialized training is not required. With respect to the generalizability beyond synchronous behavior, it is worth noting that the current musical paradigm has been utilized in previous studies exploring self-other distinction (Novembre et al., 2012), coordination (Novembre et al., 2014), and turn taking (Hadley et al., 2015). The latter two studies, demonstrate not only that pianists rely on internal motor representations in the context of both synchronous and turn-taking interactions, but also that these representations play a causal role in supporting coordination because interfering with them using repetitive transcranial magnetic stimulation impairs inter-personal coordination accuracy (Novembre et al., 2014, Hadley et 
al., 2015). Whether this conclusion holds for non-musical turn taking is a topic of interest (Pickering and Garrod 2007, 2013, 2014) that deserves to be explored.

\subsection{Alpha oscillations are sensitive to millisecond timescale variations in interpersonal coordination.}

Consistent with previous research on relations between interpersonal coordination and neural oscillations (see Babiloni and Astolfi 2014 for a review), we found that EEG alpha power over centro-parietal scalp regions was generally more suppressed with higher self-other entrainment. It is remarkable that these alpha modulations were observed with subtle changes in behavioral self-other synchronization and mutual adaptation at the millisecond timescale. Our findings therefore suggest that alpha oscillations are not only associated with large-scale changes in interpersonal coordination, such as those occurring in interactive vs. non-interactive tasks (Tognoli et al. 2007; Konvalinka et al. 2014) or with in-phase vs. anti-phase dyadic movements (Naeem et al. 2012a, 2012b). Alpha oscillatory activity may thus partially reflect highly resolved error correction mechanisms (Repp 2005; Repp and Su 2013) that operate at the millisecond time scale to support the precise synchronization of self-generated movements with external rhythms in the context of social coordination tasks.

\subsection{Theoretical advance and implications}

From a broader perspective, the interaction of co-representation and self-other entrainment indexed by alpha oscillations provides a new conceptualization of 
the link between physical (external) and psychological (internal) dimensions of social cognition. While the focus of entrainment is on the external physical environment, specifically, the relationship between dynamically changing sensory input and emergent motor responses (Gibson 1979; Turvey 1990; Shaw 2001), co-representation is focused more internally on the retrieval of acquired knowledge that provides a perspective on others 'from the inside' (cf. Rizzolatti and Sinigaglia 2010; Casile et al. 2011; Gallese 2014). Importantly, here we show that alpha oscillations play a role in regulating the balance between self-other integration and segregation depending on the compatibility of internal and external information. Physical and psychological dimensions are thus linked when events observed in the surrounding environment are compatible with internal knowledge.

From a computational perspective, it is useful to frame the concept of varying compatibility between internal and external information in terms of predictive accounts of mirroring mechanisms (Wilson and Knoblich 2005; Kilner et al. 2007; Keysers et al. 2014; Rizzolatti and Fogassi 2014), as indexed by oscillations in the alpha range (Arnstein et al. 2011). In the context of joint action, mirroring mechanisms have been implicated in the process of generating mutual predictions between collaborating individuals (cf. Friston and Frith 2015). Such accounts would hold that predictions aimed at minimizing surprise about other's behavior (Friston 2010) are informed by both internal knowledge and self-other entrainment during real-time interpersonal coordination. It follows that agreement or conflict between internally and externally guided predictions would result in self-other integration and segregation, respectively. 
In sum, we suggest that alpha oscillations constitute a promising neural marker indexing the interaction between environmental (external) and psychological (internal) sources of information in the context of real-time human interaction. Specifically, we contend that alpha oscillations balance between integration and segregation of internally and externally driven information, or predictions, concerning self- and other-related actions and effects.

\section{Acknowledgments}

We thank Katarzyna Gugnowska, Roberta Bianco, Heike Boethel, Kerstin Traeger and Thomas Gunter for their help and support with data collection, Sven Gutekunst and Jan Bergmann for technical support, Maren Grigutsch, Freek van Ede and Manuel Varlet for useful comments and discussions, and the pianists who took part in the study. This research was supported by the Max Planck Society.

\section{REFERENCES}

Acquadro MAS, Congedo M, De Riddeer D. 2016. Music Performance As an Experimental Approach to Hyperscanning Studies. Front Hum Neurosci. 10:1-13.

Aglioti SM, Cesari P, Romani M, Urgesi C. 2008. Action anticipation and motor resonance in elite basketball players. Nat Neurosci. 11:1109-1116.

Arnstein D, Cui F, Keysers C, Maurits NM, Gazzola V. 2011. -Suppression during Action Observation and Execution Correlates with BOLD in Dorsal Premotor, Inferior Parietal, and SI Cortices. J Neurosci. 31:14243-14249.

Babiloni F, Astolfi L. 2014. Social neuroscience and hyperscanning techniques: 
Past, present and future. Neurosci Biobehav Rev. 44:76-93.

Battelli L, Pascual-Leone A, Cavanagh P. 2007. The "when" pathway of the right parietal lobe. Trends Cogn Sci. 11:204-210.

Bazanova OM, Vernon D. 2013. Interpreting EEG alpha activity. Neurosci Biobehav Rev. 44:94-110.

Beckes L, Coan J a., Hasselmo K. 2013. Familiarity promotes the blurring of self and other in the neural representation of threat. Soc Cogn Affect Neurosci. 8:670-677.

Bekkering H, de Bruijn ER a., Cuijpers RH, Newman-Norlund R, van Schie HT, Meulenbroek R. 2009. Joint Action: Neurocognitive Mechanisms Supporting Human Interaction. Top Cogn Sci. 1:340-352.

Benedek M, Schickel RJ, Jauk E, Fink A, Neubauer AC. 2014. Alpha power increases in right parietal cortex reflects focused internal attention. Neuropsychologia. 56:393-400.

Brass M, Derrfuss J, von Cramon DY. 2005. The inhibition of imitative and overlearned responses: a functional double dissociation. Neuropsychologia. 43:89-98.

Brass M, Ruby P, Spengler S. 2009. Inhibition of imitative behaviour and social cognition. Philos Trans R Soc Lond B Biol Sci. 364:2359-2367.

Cacioppo S, Zhou H, Monteleone G, Majka E a, Quinn K a, Ball AB, Norman GJ, Semin GR, Cacioppo JT. 2014. You are in sync with me: Neural correlates of interpersonal synchrony with a partner. Neuroscience.

Caetano G, Jousmäki V, Hari R. 2007. Actor's and observer's primary motor cortices stabilize similarly after seen or heard motor actions. Proc Natl Acad Sci U S A.

Calvo-Merino B, Glaser DE, Grèzes J, Passingham RE, Haggard P. 2005. Action observation and acquired motor skills: an FMRI study with expert dancers. Cereb Cortex. 15:1243-1249.

Calvo-Merino B, Grèzes J, Glaser DE, Passingham RE, Haggard P. 2006. Seeing or doing? Influence of visual and motor familiarity in action observation. Curr Biol. 16:1905-1910.

Cannon EN, Yoo KH, Vanderwert RE, Ferrari PF, Woodward AL, Fox N a. 2014. Action experience, more than observation, influences mu rhythm desynchronization. PLoS One. 9:1-8.

Casile a., Caggiano V, Ferrari PF. 2011. The Mirror Neuron System: A Fresh View. Neurosci. 17:524-538.

Cirelli LK, Einarson KM, Trainor LJ. 2014. Interpersonal synchrony increases prosocial behavior in infants. Dev Sci. 1-9.

Clayton M, Sager R, Will U. 2005. In time with the music: the concept of entrainment and its significance for ethnomusicology. Eur Meet Ethnomusicol. 11:1-45. 
Coey C a, Varlet M, Richardson MJ. 2012. Coordination dynamics in a socially situated nervous system. Front Hum Neurosci. 6:164.

Colling LJ, Williamson K. 2014. Entrainment and motor emulation : Alternatives or complementary approaches ? 8:1-11.

Colzato LS, de Bruijn ER a, Hommel B. 2012. Up to "me" or up to "us"?The impact of self-construal priming on cognitive self-other integration. Front Psychol. 3:1-4.

Cummins F. 2014. Voice, (inter-)subjectivity, and real time recurrent interaction. Front Psychol. 5.

D’Ausilio A, Altenmüller E, Olivetti Belardinelli M, Lotze M. 2006. Cross-modal plasticity of the motor cortex while listening to a rehearsed musical piece. Eur J Neurosci. 24:955-958.

D'Ausilio A, Novembre G, Fadiga L, Keller PE. 2015. What can music tell us about social interaction? Trends Cogn Sci. 19:1-4.

Decety J, Lamm C. 2007. The role of the right temporoparietal junction in social interaction: how low-level computational processes contribute to metacognition. Neuroscientist. 13:580-593.

Dolk T, Hommel B, Colzato LS, Schütz-Bosbach S, Prinz W, Liepelt R. 2014. The joint Simon effect: a review and theoretical integration. Front Psychol. 5:974.

Dumas G, Martinerie J, Soussignan R, Nadel J. 2012. Does the brain know who is at the origin of what in an imitative interaction? Front Hum Neurosci. 6:128.

Dumas G, Nadel J, Soussignan R, Martinerie J, Garnero L. 2010. Inter-brain synchronization during social interaction. PLoS One. 5:e12166.

Dumas G, Soussignan R, Hugueville L, Martinerie J, Nadel J. 2014. Revisiting mu suppression in autism spectrum disorder. Brain Res. 1-12.

Fairhurst MT, Janata P, Keller PE. 2013. Being and feeling in sync with an adaptive virtual partner: brain mechanisms underlying dynamic cooperativity. Cereb Cortex. 23:2592-2600.

Fairhurst MT, Janata P, Keller PE. 2014. Leading the follower: an fMRI investigation of dynamic cooperativity and leader-follower strategies in synchronization with an adaptive virtual partner. Neuroimage. 84:688-697.

Frenkel-Toledo S, Bentin S, Perry A, Liebermann DG, Soroker N. 2014. Mirrorneuron system recruitment by action observation: Effects of focal brain damage on mu suppression. Neuroimage. 87:127-137.

Friston K. 2010. The free-energy principle: a unified brain theory? Nat Rev Neurosci. 11:127-138.

Friston K, Frith C. 2015. A Duet for one. Conscious Cogn.

Gallese V. 2014. Bodily selves in relation: embodied simulation as second-person perspective on intersubjectivity. Philos Trans R Soc Lond B Biol Sci. 369:20130177. 
Gastaut, H. J., \& Bert J. 1954. EEG changes during cinematographic presentation (Moving picture activation of the EEG). Electroencephalogr Clin Neurophysiol. 6:433-444.

Gibson JJ. 1979. The ecological approach to visual perception, of Experimental Psychology Human Perception and.

Goebl W, Palmer C. 2009. Synchronization of timing and motion among performing musicians. Music Percept. 26:427-438.

Greenfield MD. 1994. Cooperation and Conflict in the Evolution of Signal Interactions. Annu Rev Ecol Syst.

Hadley L V, Novembre G, Keller PE, Pickering MJ. 2015. Causal Role of Motor Simulation in Turn-Taking Behavior. J Neurosci. 35:16516-16520.

Haegens S, Osipova D, Oostenveld R, Jensen O. 2010. Somatosensory working memory performance in humans depends on both engagement and disengagement of regions in a distributed network. Hum Brain Mapp. 31:26-35.

Hari R, Forss N, Avikainen S, Kirveskari E, Salenius S, Rizzolatti G. 1998. Activation of human primary motor cortex during action observation: a neuromagnetic study. Proc Natl Acad Sci U S A. 95:15061-15065.

Hove MJ, Risen JL. 2009. It's All in the Timing: Interpersonal Synchrony Increases Affiliation. Soc Cogn. 27:949-960.

Jensen O, Bonnefond M, VanRullen R. 2012. An oscillatory mechanism for prioritizing salient unattended stimuli. Trends Cogn Sci. 16:200-205.

Jensen 0, Mazaheri A. 2010. Shaping functional architecture by oscillatory alpha activity: gating by inhibition. Front Hum Neurosci. 4:186.

Kaplan DM, Bechtel W. 2011. Dynamical models: An alternative or complement to mechanistic explanations? Top Cogn Sci. 3:438-444.

Keller PE. 2012. Mental Imagery in Music Performance: Underlying Mechanisms and Potential Benefits. Ann N Y Acad Sci.

Keller PE. 2014. Ensemble performance : Interpersonal alignment of musical expression, Expressiveness in music performance: Empirical approaches across styles and cultures.

Keller PE, Knoblich G, Repp BH. 2007. Pianists duet better when they play with themselves: on the possible role of action simulation in synchronization. Conscious Cogn. 16:102-111.

Keller PE, Novembre G, Hove MJ. 2014. Rhythm in joint action: Psychological and neurophysiological mechanisms for real-time interpersonal coordination. Philos Trans R Soc Lond B Biol Sci.

Keller PE., Novembre G., Loehr J. 2016. Musical ensemble performance: Representing self, other, and joint action outcomes. In: Cross; E.,, Obhi; S., editors. Shared representations: Sensorimotor foundations of social life. Cambridge, MA: Cambridge University Press. 
Keysers C, Gazzola V, B PTRS. 2014. Hebbian learning and predictive mirror neurons for actions, sensations and emotions. Philos Trans R Soc B. 369:20130175.

Kilner JM, Friston KJ, Frith CD. 2007. The mirror-neuron system: a Bayesian perspective. Neuroreport. 18:619-623.

Kirschner S, Tomasello M. 2010. Joint music making promotes prosocial behavior in 4-year-old children. Evol Hum Behav. 31:354-364.

Klimesch W. 1997. EEG-alpha rhythms and memory processes. Int J Psychophysiol. 26:319-340.

Klimesch W. 2012. Alpha-band oscillations, attention, and controlled access to stored information. Trends Cogn Sci. 16:606-617.

Klimesch W, Sauseng P, Hanslmayr S. 2007. EEG alpha oscillations: the inhibition-timing hypothesis. Brain Res Rev. 53:63-88.

Knoblich G, Sebanz N. 2008. Evolving intentions for social interaction: from entrainment to joint action. Philos Trans R Soc Lond B Biol Sci. 363:20212031.

Kokal I, Engel A, Kirschner S, Keysers C. 2011. Synchronized Drumming Enhances Activity in the Caudate and Facilitates Prosocial Commitment-If the Rhythm Comes Easily. PLoS One. 6:e27272.

Konvalinka I, Bauer M, Stahlhut C, Hansen LK, Roepstorff A, Frith CD. 2014. Frontal alpha oscillations distinguish leaders from followers: Multivariate decoding of mutually interacting brains. Neuroimage.

Konvalinka I, Vuust P, Roepstorff A, Frith CD. 2010. Follow you, follow me: Continuous mutual prediction and adaptation in joint tapping. Q J Exp Psychol (Hove). 1-11.

Krampe RT, Engbert R, Kliegl R. 2002. Representational models and nonlinear dynamics: irreconcilable approaches to human movement timing and coordination or two sides of the same coin? Introduction to the special issue on movement timing and coordination. Introduction. Brain Cogn. 48:1-6.

Lahav A, Saltzman E, Schlaug G. 2007. Action representation of sound: audiomotor recognition network while listening to newly acquired actions. J Neurosci. 27:308-314.

Large EW. 2008. Resonating to musical rhythm: theory and experiment, Psychology of time.

Lee H, Noppeney U. 2011. Long-term music training tunes how the brain temporally binds signals from multiple senses. Proc Natl Acad Sci U S A. 2011:1-10.

Liepelt R, Schneider JC, Aichert DS, Wöstmann N, Dehning S, Möller HJ, Riedel M, Dolk T, Ettinger U. 2012. Action blind: Disturbed self-other integration in schizophrenia. Neuropsychologia. 50:3775-3780.

Loehr JD, Kourtis D, Vesper C, Sebanz N, Knoblich G. 2013. Monitoring individual 
and joint action outcomes in duet music performance. J Cogn Neurosci. 25:1049-1061.

Loehr JD, Vesper C. 2015. The sound of you and me: Novices represent shared goals in joint action. Q J Exp Psychol (Hove). 69:535-547.

Maris E, Oostenveld R. 2007. Nonparametric statistical testing of EEG- and MEGdata. J Neurosci Methods. 164:177-190.

Marsh KL, Isenhower RW, Richardson MJ, Helt M, Verbalis AD, Schmidt RC, Fein D. 2013. Autism and social disconnection in interpersonal rocking. Front Integr Neurosci. 7:4.

Marsh KL, Richardson MJ, Baron RM, Schmidt RC. 2006. Contrasting Approaches to Perceiving and Acting With Others. Ecol Psychol.

McGarry T, Anderson DI, Wallace S a, Hughes MD, Franks IM. 2002. Sport competition as a dynamical self-organizing system. J Sports Sci. 20:771-781.

Meeuwissen EB, Takashima A, Fernández G, Jensen 0. 2011. Increase in posterior alpha activity during rehearsal predicts successful long-term memory formation of word sequences. Hum Brain Mapp. 32:2045-2053.

Merker B. 2000. Synchronous Chorusing and Human Origins, The Origins of Music.

Merker BH, Madison GS, Eckerdal P. 2009. On the role and origin of isochrony in human rhythmic entrainment. Cortex. 45:4-17.

Meyer M, Hunnius S, van Elk M, van Ede F, Bekkering H. 2011. Joint action modulates motor system involvement during action observation in 3-yearolds. Exp Brain Res. 211:581-592.

Mithen S, Morley I, Wray A, Tallerman M, Gamble C. 2006. The Singing Neanderthals: the Origins of Music, Language, Mind and Body , by Steven Mithen. London: Weidenfeld \& Nicholson, 2005. Cambridge Archaeol J.

Naeem M, Prasad G, Watson DR, Kelso J a S. 2012a. Electrophysiological signatures of intentional social coordination in the $10-12 \mathrm{~Hz}$ range. Neuroimage. 59:1795-1803.

Naeem M, Prasad G, Watson DR, Kelso J a S. 2012b. Functional dissociation of brain rhythms in social coordination. Clin Neurophysiol. 123:1789-1797.

Naeem M, Prasad G, Watson DR, Kelso J a S. 2012c. Electrophysiological signatures of intentional social coordination in the $10-12 \mathrm{~Hz}$ range. Neuroimage. 59:1795-1803.

Newman-Norlund RD, van Schie HT, van Zuijlen AMJ, Bekkering H. 2007. The mirror neuron system is more active during complementary compared with imitative action. Nat Neurosci. 10:817-818.

Nobre AC. 2001. Orienting attention to instants in time. Neuropsychologia. 39:1317-1328.

Novembre G, Keller PE. 2014. A conceptual review on action-perception coupling in the musicians' brain: what is it good for? Front Hum Neurosci. 8:1-11. 
Novembre G, Ticini LF, Schutz-Bosbach S, Keller PE. 2014. Motor simulation and the coordination of self and other in real-time joint action. Soc Cogn Affect Neurosci. 9:1062-1068.

Novembre G, Ticini LF, Schütz-Bosbach S, Keller PE. 2012. Distinguishing self and other in joint action. Evidence from a musical paradigm. Cereb Cortex. 22:2894-2903.

Obleser J, Weisz N. 2012. Suppressed alpha oscillations predict intelligibility of speech and its acoustic details. Cereb Cortex. 22:2466-2477.

Oostenveld R, Fries P, Maris E, Schoffelen JM. 2011. FieldTrip: Open source software for advanced analysis of MEG, EEG, and invasive electrophysiological data. Comput Intell Neurosci. 2011.

Paladino M-P, Mazzurega M, Pavani F, Schubert TW. 2010. Synchronous multisensory stimulation blurs self-other boundaries. Psychol Sci a J Am Psychol Soc / APS. 21:1202-1207.

Phillips-Silver J, Keller PE. 2012. Searching for roots of entrainment and joint action in early musical interactions. Front Hum Neurosci. 6:26.

Pickering MJ, Garrod S. 2007. Do people use language production to make predictions during comprehension? Trends Cogn Sci. 11:105-110.

Pickering MJ, Garrod S. 2013. An integrated theory of language production and comprehension. Behav Brain Sci. 36:329-347.

Pickering MJ, Garrod S. 2014. Neural integration of language production and comprehension. Proc Natl Acad Sci. 111:15291-15292.

Pressing J. 1999. The referential dynamics of cognition and action. Psychol Rev.

Prinz W. 1997. Perception and Action Planning. Eur J Cogn Psychol. 9:129-154.

Prinz W. 2013. Self in the mirror. Conscious Cogn. 22:1105-1113.

Quandt LC, Marshall PJ. 2014. The effect of action experience on sensorimotor EEG rhythms during action observation. Neuropsychologia. 56:401-408.

Ragert M, Schroeder T, Keller PE. 2013. Knowing too little or too much: the effects of familiarity with a co-performer's part on interpersonal coordination in musical ensembles. Front Psychol. 4:368.

Repp B, Keller PE. 2004. Adaptation to tempo changes in sensorimotor synchronization: Effects of intention, attention, and awareness. Q J Exp. 57a:499-521.

Repp BH. 2001. Processes underlying adapation to tempo changes in sensorimotor synchronisation. Hum Mov Sci.

Repp BH. 2005. Sensorimotor synchronization: A review of the tapping literature. Psychon Bull \&amp; Rev. 12:969-992.

Repp BH, Su Y-H. 2013. Sensorimotor synchronization: a review of recent research (2006-2012). Psychon Bull Rev. 20:403-452.

Richardson MJ, Marsh KL, Isenhower RW, Goodman JRL, Schmidt RC. 2007. 
Rocking together: Dynamics of intentional and unintentional interpersonal coordination. Hum Mov Sci. 26:867-891.

Rizzolatti G, Fogassi L. 2014. The mirror mechanism: recent findings and perspectives. Philos Trans R Soc Lond B Biol Sci. 369:20130420.

Rizzolatti G, Sinigaglia C. 2010. The functional role of the parieto-frontal mirror circuit: interpretations and misinterpretations. Nat Rev Neurosci. 11:264274.

Rohenkohl G, Nobre a. C. 2011. Alpha Oscillations Related to Anticipatory Attention Follow Temporal Expectations. J Neurosci. 31:14076-14084.

Ruby P, Decety J. 2001. Effect of subjective perspective taking during simulation of action: a PET investigation of agency. Nat Neurosci. 4:546-550.

Salmelin R, Hari R. 1994. Spatiotemporal characteristics of sensorimotor neuromagnetic rhythms related to thumb movement. Neuroscience. 60:537-550.

Samaha J, Bauer P, Cimaroli S, Postle BR. 2015. Top-down control of the phase of alpha-band oscillations as a mechanism for temporal prediction. Proc Natl Acad Sci. 112:8439-8444.

Santiesteban I, Banissy MJ, Catmur C, Bird G. 2012. Enhancing social ability by stimulating right temporoparietal junction. Curr Biol. 22:2274-2277.

Sauseng P, Klimesch W, Gerloff C, Hummel FC. 2009. Spontaneous locally restricted EEG alpha activity determines cortical excitability in the motor cortex. Neuropsychologia. 47:284-288.

Schiavio A, Hoffding S. 2015. Playing together without communicating? A prereflective and enactive account of joint musical performance. Music Sci. 19:366-388.

Schilbach L. 2010. A second-person approach to other minds. Nat Rev Neurosci. 11:449.

Schmidt RC, Carello C, Turvey MT. 1990. Phase transitions and critical fluctuations in the visual coordination of rhythmic movements between people. J Exp Psychol Hum Percept Perform. 16:227-247.

Schmidt RC, Fitzpatrick P, Caron R, Mergeche J. 2011. Understanding social motor coordination. Hum Mov Sci. 30:834-845.

Sebanz N, Bekkering H, Knoblich G. 2006. Joint action: bodies and minds moving together. Trends Cogn Sci. 10:70-76.

Sebanz N, Knoblich G, Prinz W. 2003. Representing others' actions: just like one's own? Cognition. 88:11-21.

Sebanz N, Knoblich G, Prinz W, Wascher E. 2006. Twin peaks: an ERP study of action planning and control in co-acting individuals. J Cogn Neurosci. 18:859-870.

Sharbrough, F., Chatrian, G. E., Lesser, R. P., Lüders, H., Nuwer, M., \& Picton TW. 1991. American Electroencephalographic Society guidelines for standard 
electrode position nomenclature. J clin Neurophysiol,. 8:200-202.

Shaw R. 2001. Processes, Acts, and Experiences: Three Stances on the Problem of Intentionality. Ecol Psychol.

Stepp N, Chemero A, Turvey MT. 2011. Philosophy for the rest of cognitive science. Top Cogn Sci. 3:425-437.

Tamura Y, Hoshiyama M, Nakata H, Hiroe N, Inui K, Kaneoke Y, Inoue K, Kakigi R. 2005. Functional relationship between human rolandic oscillations and motor cortical excitability: An MEG study. Eur J Neurosci. 21:2555-2562.

Thut G, Nietzel A, Brandt S a, Pascual-Leone A. 2006. Alpha-band electroencephalographic activity over occipital cortex indexes visuospatial attention bias and predicts visual target detection. J Neurosci. 26:94949502.

Tognoli E, Kelso J a S. 2014. The metastable brain. Neuron. 81:35-48.

Tognoli E, Lagarde J, DeGuzman GC, Kelso J a S. 2007. The phi complex as a neuromarker of human social coordination. Proc Natl Acad Sci U S A. 104:8190-8195.

Tomasello M, Carpenter M, Call J, Behne T, Moll H. 2005. Understanding and sharing intentions: the origins of cultural cognition. Behav Brain Sci. 28:675-691.

Tsakiris M, Costantini M, Haggard P. 2008. The role of the right temporo-parietal junction in maintaining a coherent sense of one's body. Neuropsychologia. 46:3014-3018.

Turvey MT. 1990. Coordination. Am Psychol. 45:938-953.

Urgesi C, Maieron M, Avenanti A, Tidoni E, Fabbro F, Aglioti SM. 2010. Simulating the future of actions in the human corticospinal system. Cereb Cortex. 20:2511-2521.

van der Steen MC, Jacoby N, Fairhurst MT, Keller PE. 2015. Sensorimotor synchronization with tempo-changing auditory sequences: Modeling temporal adaptation and anticipation. Brain Res. 1-22.

van der Steen MCM, Keller PE. 2013. The ADaptation and Anticipation Model (ADAM) of sensorimotor synchronization. Front Hum Neurosci. 7:253.

van Ede F, de Lange F, Jensen O, Maris E. 2011. Orienting attention to an upcoming tactile event involves a spatially and temporally specific modulation of sensorimotor alpha- and beta-band oscillations. J Neurosci. 31:2016-2024.

van Ede F, Jensen O, Maris E. 2010. Tactile expectation modulates pre-stimulus beta-band oscillations in human sensorimotor cortex. Neuroimage. 51:867876.

van Elk M, van Schie HT, Hunnius S, Vesper C, Bekkering H. 2008. You'll never crawl alone: neurophysiological evidence for experience-dependent motor resonance in infancy. Neuroimage. 43:808-814. 
van Ulzen NR, Lamoth CJC, Daffertshofer A, Semin GR, Beek PJ. 2008.

Characteristics of instructed and uninstructed interpersonal coordination while walking side-by-side. Neurosci Lett. 432:88-93.

Varlet M, Marin L, Capdevielle D, Del-Monte J, Schmidt RC, Salesse RN, Boulenger J-P, Bardy BG, Raffard S. 2014. Difficulty leading interpersonal coordination: towards an embodied signature of social anxiety disorder. Front Behav Neurosci. 8:29.

Varlet M, Marin L, Raffard S, Schmidt RC, Capdevielle D, Boulenger J-P, Del-Monte J, Bardy BG. 2012. Impairments of social motor coordination in schizophrenia. PLoS One. 7:e29772.

Vorberg D, Wing A. 1996. Modeling variability and dependence in timing, Handbook of perception and action.

Wilson M, Knoblich G. 2005. The case for motor involvement in perceiving conspecifics. Psychol Bull. 131:460-473.

Wiltermuth SS, Heath C. 2009. Synchrony and cooperation. Psychol Sci. 20:1-5.

Wing AM, Endo S, Bradbury A, Vorberg D. 2014. Optimal feedback correction in string quartet synchronization. J R Soc Interface. 11:20131125.

Wolpert DM, Doya K, Kawato M. 2003. A unifying computational framework for motor control and social interaction. Philos Trans R Soc Lond B Biol Sci. 358:593-602.

Worden MS, Foxe JJ, Wang N, Simpson G V. 2000. Anticipatory biasing of visuospatial attention indexed by retinotopically specific alpha-band electroencephalography increases over occipital cortex. J Neurosci. 20:RC63. 